FORUM EDITORIAL

\title{
CELLULAR REDOX COMPARTMENTS
}

Thomas

Kietzmann

Faculty of Biochemistry and Molecular Medicine, and Biocenter Oulu, University of Oulu, FI-90014 Oulu, Finland;

Address for correspondence: Dr. Thomas Kietzmann,

University of Oulu

Faculty of Biochemistry and Molecular Medicine

Aapistie 7C

90230 Oulu

Finland

Tel.: +358294487713

Email: Thomas.Kietzmann@oulu.fi

Running head: Redox Compartments

Keywords: Reactive oxygen species, endoplasmic reticulum, peroxisomes, lysosomes, autophagy, NADPH oxidase, Golgi apparatus, cell organelles, protein folding, Glycobiology, oxidative stress

\begin{abstract}
Although initially considered of being harmful, reactive oxygen species (ROS) are nowadays also recognized as important signaling molecules affecting various cellular processes. For example, they contribute to the response to hormones, growth factors, or hypoxia, and defense reactions against mechanical or chemical stress. Thereby, different ROS-generators, ROS-utilizing, and ROS-degrading systems in different intracellular compartments play an important role. One the one hand, this leads to a functional specialization where proteins, which participate in a specific ROS regulated pathway in one compartment, may have another ROS-unrelated specific function in another compartment. On the other hand, this adds also a layer of protection by keeping unwanted side reactions to a minimum. Accordingly, the intracellular communication between different cellular compartments is an important mechanism to achieve effects on the cellular level. Antioxid. Redox Signal. xx, xxx-xxx.
\end{abstract}


Reactive oxygen species (ROS) are often considered as deleterious and toxic products of important oxygen using cellular reactions. Superoxide anion radical $\left(\mathrm{O}_{2}-^{-}\right)$formation is often the initial step in ROS generation, which then participates in the generation of other ROS: most important, hydrogen peroxide $\left(\mathrm{H}_{2} \mathrm{O}_{2}\right)$, hydroxyl radicals $(\mathrm{OH} \bullet)$, peroxynitrite (ONOO-), hypochlorous acid $(\mathrm{HOCl})$, and singlet oxygen $\left({ }^{1} \mathrm{O}_{2}\right)$. Excess formation of these species may result in DNA, lipid and protein damage; however, under physiological conditions this is commonly balanced by the endogenous antioxidant systems consisting of enzymes and redox couples as well as exogenously taken up micronutrients and vitamins (4).

In contrast, during the last two decades, a number of laboratories including our own have obtained ample evidence that low or moderate formation of reactive oxygen species (ROS) are also involved in various physiological responses and take part in different signaling processes and even defense reactions.

To allow proper ROS signaling and to limit deleterious side effects, ROS generation, utilization, and degradation need to be spatially restricted and strictly regulated. Eukaryotic cells, which display functional specialization due to compartmentalization into organelles, appear to employ ROSgenerating, and ROS-degrading systems within each compartment. Although this compartmentalization limits the damaging effects of ROS, it demands a high level of communication between the different cellular compartments to achieve the proper effects at the cellular level. Thereby, proteins that participate in a specific ROS regulated pathway in one compartment may have another ROS-unrelated specific function in another compartment. Moreover, this requires cooperation of the ROS relays within and between the compartments with systems specifically responding to extracellular/environmental clues such as hormones, growth factors, and mechanical or chemical stress; hence, adding another layer of complexity to the ROS signaling systems.

Since the intracompartmental communication in ROS signaling is just at the beginning to be understood, the current FORUM ISSUE, which comprises a series of review articles and original manuscripts, aimed to highlight current knowledge and important new findings related to different aspects of cell signaling in response to ROS in different cellular compartments (Fig.1).

In their review, Petry and Görlach (5) address the link from extracellular stimuli to intracellular ROS generation. Thereby, they focus on plasma membrane integrated G protein coupled receptors (GPCRs), from which members are involved in the response to light, odors, hormones, and neurotransmitters as well as in ROS generation. As the largest group of cell-surface receptors GPCRs were shown to generate ROS via a link to a family of NADPH oxidases (NOX). Although various participating components have been characterized, a more detailed analysis of $\mathrm{G}$ proteins involved and a more 
precise discrimination of NADPH oxidase activation due to either upregulation of subunit expression or post-translational subunit modifications is still needed. The understanding of GPCR-mediated NADPH oxidase activation and that of the non-canonical NADPH oxidase modulators, will provide new opportunities for improved efficiency and decreased off-target effects of GPCR targeting drugs.

While GPCR signaling and NADPH oxidase regulation is a relatively recent area of research, the two principal GPCR signal transduction pathways are the CAMP pathway and the phosphatidylinositol signal pathway. The formation of inositol 1,4,5-trisphosphate (IP3) in the latter links GPCR function also to another organelle, the endoplasmic reticulum (ER). There, IP3 binds to the IP3 receptor and opens $\mathrm{Ca}^{2+}$ channels to release $\mathrm{Ca}^{2+}$ into the cytosol. As discussed in the review by Mennerich et al. (4) this may hamper the function of NADPH oxidases since the ER itself harbors NADPH oxidase 4, -5 , and DUOX1/2 where NOX5 and DUOX1/2 are being responsive to $\mathrm{Ca}^{2+}$. The resulting change in redox homeostasis may lead to ER stress. By combining in-vitro and in-vivo experiments Petry et al. (7) found that the PERK-ATF4 branch of the ER stress response, enhanced the expression of p22phox, the cytosolic activator of the neutrophil and vascular NOX2. Hence, these data identify a feedback mechanism where ER stress can contribute to p22phox-NOX2 mediated ROS formation at the plasma membrane of neutrophils and vascular cells.

Moreover, changing ROS conditions as appearing under hypoxia as exemplified in pulmonary hypertension, appear not only to exert a transcriptional p22phox response but also to affect p22phox protein stability in vitro and in vivo. Thereby, Petry et al. (6) showed in their original contribution that hypoxia decreased ubiquitiylation and proteasomal degradation of p22phox by involving prolyl hydroxylases and the pVHL E3 ubiquitin ligase complex. The resulting p22phox stabilization and accumulation increased also the levels and activity of hypoxia-inducible factor- $1 \alpha$ possibly by decreasing the levels of the prolyl hydroxylase cofactors ascorbate and iron(II), which establishes p22phox as an important regulator of the hypoxia response.

Although hypoxia, ROS, and $\mathrm{pH}$ changes in the ER and Golgi as discussed in the review by Mennerich et al (4) are very much associated with diseases such as type 2 diabetes, Alzheimer's disease and cancer, it remained so far unknown whether any of those factors contributes to mistargeting of tumor markers from apical to basolateral membranes in polarized cells. In their original manuscript Kokkonen et al. (2) investigated whether hypoxia, ROS, or an altered Golgi $\mathrm{pH}$, can be responsible for mistargeting of carcinoembryonic antigen (CEACAM5, CEA), which is a known tumor marker for colorectal cancer. By using a number of elegant experiments, they found that in contrast to hypoxia and ROS, an abnormal Golgi pH homeostasis is an important factor that causes mistargeting of CEA from the apical membrane in normal cells to the basolateral surface of cancer cells via inhibiting its GPI-anchor-mediated association with lipid rafts. 
The generation of ROS, $\mathrm{H}_{2} \mathrm{O}_{2}$ in particular, within the ER appears not only to be relevant during protein folding processes but also in a context where the ER participates in inter-compartment signaling where the permeability of the membrane for $\mathrm{H}_{2} \mathrm{O}_{2}$ is facilitated by aquaporin channels (4). Another organelle that substantially contributes to production or breakdown of $\mathrm{H}_{2} \mathrm{O}_{2}$ are peroxisomes. However, so far the role of this organelle in cross-compartmental $\mathrm{H}_{2} \mathrm{O}_{2}$ signaling has remained elusive most likely due to technical difficulties, which did not allow manipulation of peroxisomal $\mathrm{H}_{2} \mathrm{O}_{2}$ production. The original contribution by Lismont et al (3) shows how these problems can be solved. By using a series of elaborate and elegant steps the authors developed a human cell line that can be used to selectively generate $\mathrm{H}_{2} \mathrm{O}_{2}$ inside peroxisomes in a time- and dose-controlled manner. Importantly, the authors show that peroxisome-derived $\mathrm{H}_{2} \mathrm{O}_{2}$ can oxidize proteins inside and outside of the peroxisomal compartment in a manner being inversely correlated to catalase activity. As then discussed in the review by Fransen (1), the precise molecular mechanisms underlying these observations are still quite unknown, and to better understand chronic diseases and aging, a key focus for future research should be the identification of primary peroxisomal-generated $\mathrm{H}_{2} \mathrm{O}_{2}$ targets.

$\mathrm{H}_{2} \mathrm{O}_{2}$ generation and signaling is very much influenced by the NADH/NAD+ and NADPH/NADP+ ratio. In addition to NADPH oxidases also other enzymes such as sirtuins can be affected and it has been shown that NAD+ activates sirtuins. In mammals seven sirtuins (SIRT1-7) in different compartments have been described. While SIRT1, -2, -6, -7 are found in the nucleus, SIRT1 and SIRT2 are cytoplasmic proteins and SIRT3, -4 and -5 are localized in the mitochondria. A lot of emphasis has been put on the regulation of SIRT1 and accordingly, the original contribution by Rada et al. (8) shows that SIRT1 is a key player in the pro-inflammatory and oxidative stress response that can protect the liver from acetaminophen (APAP)-mediated toxicity. In vitro and in-vivo experiments revealed that APAP intoxication negatively regulated SIRT1 levels via interleukin $1 \beta$ and nuclear factor kappa $B$ (NFKB) signaling. Consequently, maintaining adequate SIRT1 levels might be clinically relevant in situations associated with liver toxicity. In addition, the modulation of SIRT1 activity via the NADH/NAD+ ratio has also an impact on autophagy.

Autophagy is a homeostatic process that enables degradation and turnover of substrates via membranedependent sequestration in autophagic vesicles (autophagosomes) and subsequent lysosomal delivery of cargo. The lysosomal acidic milieu and the high concentration of thiols therein allow that low-mass iron and copper that are liberated after degradation of metalloproteins feed redox reactions; hence, lysosomes are also a redox-active compartment. Accordingly, in their review Ryter et al. (9) discuss that autophagy, as a lysosome-dependent process, is highly regulated by oxidative stress, including exposure to altered oxygen tension. Vice versa, autophagy also contributes to the defenses against oxidative stress. With respect to the latter and the role of mitochondria as a major source of ROS, a 
selective form of autophagy targeting the mitochondria (mitophagy) is of critical importance. Since mitochondrial proteins are especially exposed to oxidative modification, and elimination of oxidized proteins and lipids is crucial to keep this organelle intact, mitophagy maintains mitochondrial integrity under conditions of oxidative stress. In addition, they highlight the links of autophagy with other cellular processes and diseases, which are associated with an impaired ROS homeostasis such as cardiovascular and metabolic diseases, as well as disturbances of the neural systems.

Altogether, the above-mentioned reports in conjunction with many more valuable contributions in the field, which could not been cited here due to space limitations, have contributed to the progress in our understanding about the role of ROS as signaling molecules in the different cellular compartments. While findings indicating for example the interplay between hypoxia, ER stress and Golgi function clearly indicate the intricate balance and importance of intercompartmental communication, the detailed molecular mechanisms and participating key players remain so far elusive. Thus, we are still far away from having a complete consistent picture with respect to ROS actions in and between the intracellular compartments, and a number of mechanistic details await further investigations. Resolution of these and other issues, will not only help to better understand the etiology of pathologies such as cardiovascular diseases, type 2 diabetes, Alzheimer's disease or cancer which are associated with ROS, protein aggregation, and glycosylation and autophagy defects but also to find new therapeutic options. I hope that the contributions in this Forum Issue will help to achieve this, not only by stimulating insiders but also by attracting new people to the field.

\section{Acknowledgments}

The work in the author's laboratory is supported by funds from the Finnish Academy of Science (SA296027), the Jane and Aatos Erkko Foundation, Biocenter Oulu, and the University of Oulu. 


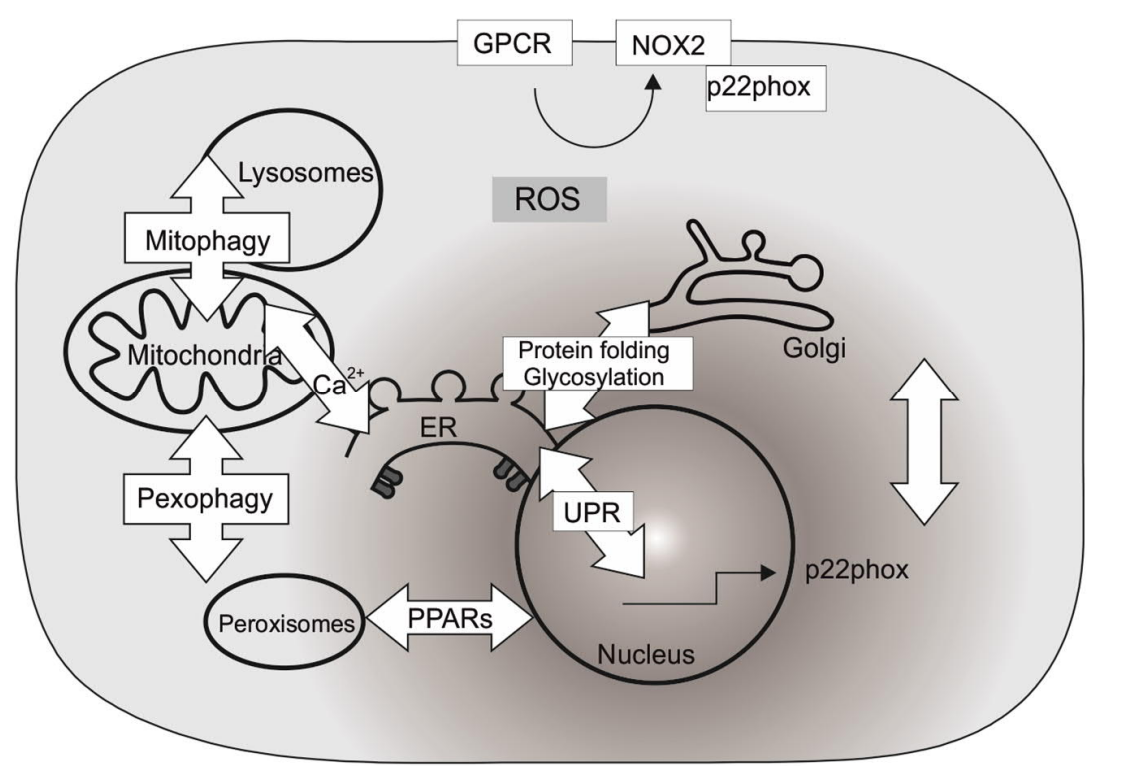

\section{Legends to figures}

Fig.1 Communication of intracellular compartments in processes involving ROS. Various intracellular compartments communicate either via ROS or in ROS involving reactions to regulate cellular adaptation processes. For example G-protein coupled receptors (GPCRs) can contribute to ROS formation via NADPH oxidases (NOX) and oxidative stress can increase p22phox expression and ROS via a feedback loop involving the UPR. ROS in the ER and Golgi are also involved in protein folding and glycosylation, as well as in the release of $\mathrm{Ca}^{2+}$ from the ER and mitochondria. ROS also contribute to selective autophagic processes such as mitophagy and pexophagy. (See the Forum article for further details)

\section{References}

1. Fransen M and Lismont C. Redox Signaling from and to Peroxisomes: Progress, Challenges, and Prospects. Antioxid Redox Signal, 2018.

2. Kokkonen N, Khosrowabadi E, Hassinen A, Harrus D, Glumoff T, Kietzmann T and Kellokumpu S. Abnormal Golgi pH Homeostasis in Cancer Cells Impairs Apical Targeting of Carcinoembryonic Antigen by Inhibiting Its Glycosyl-Phosphatidylinositol Anchor-Mediated Association with Lipid Rafts. Antioxid Redox Signal, 2018.

3. Lismont C, Nordgren M, Brees C, Knoops B, Van Veldhoven PP and Fransen M. Peroxisomes as Modulators of Cellular Protein Thiol Oxidation: A New Model System. Antioxid Redox Signal, 2017.

4. Mennerich D, Kellokumpu S and Kietzmann T. Hypoxia and Reactive Oxygen Species as Modulators 
of Endoplasmic Reticulum and Golgi Homeostasis. Antioxid Redox Signal, 2018.

5. Petry A and Gorlach A. Regulation of NADPH Oxidases by G Protein-Coupled Receptors. Antioxid Redox Signal, 2018.

6. Petry A, Trautz B, Kracun D, Zhang Z, Vogel F, Weitnauer M, Hochkogler K and Gorlach A. Stabilization of p22phox by hypoxia promotes pulmonary hypertension. Antioxid Redox Signal, 2018.

7. Petry A, Zhang Z, Trautz B, Riess F and Gorlach A. Cross Talk Between p22phox and ATF4 in the Endothelial Unfolded Protein Response. Antioxid Redox Signal, 2018.

8. Rada P, Pardo V, Mobasher MA, Garcia-Martinez I, Ruiz L, Gonzalez-Rodriguez A, Sanchez-Ramos C, Muntane J, Alemany S, James LP, Simpson KJ, Monsalve M, Valdecantos MP and Valverde AM. SIRT1 Controls Acetaminophen Hepatotoxicity by Modulating Inflammation and Oxidative Stress. Antioxid Redox Signal 28: 1187-1208, 2018.

9. Ryter SW, Bhatia D and Choi ME. Autophagy: A Lysosome-Dependent Process with Implications in Cellular Redox Homeostasis and Human Disease. Antioxid Redox Signal, 2018. 